\title{
A multilevel hypernetworks approach to assess coordination and communication in player interactions in sports teams as co-evolutionary networks
}

\author{
JOÃO RIBEIRO'1 I JÚLIO GARGANTA¹ I KEITH DAVIDS² I DANIEL BARREIRA
}

${ }^{1}$ Centre of Research, Education, Innovation and Intervention in Sport, Faculdade de Desporto, Universidade do Porto, Porto, Portugal.

2 Sheffield Hallam University, Broomgrove Teaching Block, Sheffield, United Kingdom.

Correspondence to: João Ribeiro. CIFI2D, Centre of Research, Education, Innovation and Intervention in Sport, Faculdade de Desporto, Universidade do Porto, Rua Dr. Plácido Costa, 91, 4200-450, Porto, Portugal.

email: joaoribeiro@fade.up.pt

https://doi.org/10.20338/bjmb.v14i5.216

PUBLICATION DATA

Received 09102020

Accepted 22112020

Published 01122020

\begin{abstract}
BACKGROUND: This paper presents an introduction and brief appraisal of the use of hyper networks metrics and its potential practical application in examining team dynamics' coordination patterns collective sports. AIM: Throughout their critique piece, we highlighted that game analysis, including the hyper network concept, may help overcome the limitations of previous tools such as social network measures.

FINDINGS AND CONCLUSIONS: While the social network analysis generally considers only dyadic interactions (e.g., between two players), the hyper networks also take into account a multidimensional perspective, including both player level and team level communication and coordination. We also evidenced that new studies using hyper network metrics are required in a range of team sports, mainly using data gathered from official competition matches.
\end{abstract}

A central tenet of sports science research, and, more specifically, in performance analysis, is to understand how players in team sports communicate among themselves in order to attain shared performance goals (e.g., scoring a goal). Coordination and communication represent two key processes that underpin successful performance interactions of collaborating individuals during competitive team games.

Despite a clear differentiation of roles and functions within a sports team satisfying task demands during competition requires from players the ability to undertake coordinated and functional behaviours, through communicating and interacting dynamically, interdependently, and adaptively to achieve common performance goals. ${ }^{1}$ Conceptually, like any collaborating groups, sports teams can be regarded as adaptive, co-evolutionary networks, ${ }^{2}$ with bi-directional relations between system components, expressed at higher complexity levels (micro-meso-macro relations) that are key for system functioning. ${ }^{3}$ Coordination of actions among team players during competition is determined by information communicated effectively and efficiently to support networks of interactions established between individual players or sub-groups.

Networks facilitate interactions which are key for system self-regulation and organization as they enable effective and efficient coordination and communication processes among the elements composing the system under varying circumstances (e.g., players rotating positions on field, emergence of team passing networks, covering space together). To perform these collaborative tasks, players develop local interpersonal synergies (often self-organising themselves (adjusting positions)) based on local rules predicated on perception of information about interpersonal distances, approaching velocities of teammates and opponents, ball location, and angles between teammates and opponents, etc. To exemplify, players need this information to interact with their nearest

\begin{tabular}{l|l|l|l|l}
\hline Ribeiro et al. & 2020 & VOL.14 & N.5 & https://doi.org/10.20338/bjmb.v14i5.216
\end{tabular}


teammates and/or opponents at a lower system level (micro-meso level - e.g., recovering ball-possession through a tackle in the centre corridor of the field), to generate more global, complex tactical and strategical patterns of team behaviour at a higher level (macro-level e.g., increasing interpersonal distances both in length and width to get the ball out of the pressure zone).

Of fundamental importance is that the multilevel structure and dynamics expressed at a lower level (e.g., individual player's dynamics) constrains and are constrained by the dynamics of higher-levels (e.g., the dynamics of whole team behaviour). Such interplay between dynamics of the individual players or sub-groups and the dynamics of whole team behaviour (i.e., network topology or structure) comprise a paramount feature of evolving networks inherently associated with successful performance. Evolving networks signify that the network topology (e.g., the number and shape of players' tactical configurations - e.g., players arranged in a triangular formation on-field) changes as a function of time, whilst dynamics of players also change in time. Thus, revealing the patterns and nature of the properties underlying the dynamics and structure of networks is fundamental for understanding player interactions, and understanding how tactical formations and strategies can emerge during competitive performance.

Typically, these collaborative, dynamical processes have been analysed through social network analytics that seek to shed light on system structural properties. ${ }^{4}$ Nonetheless, an important weakness of the application of these tools is that they are limited to analysis of binary relations (two-element interactions). ${ }^{5-6}$ Recent advances in technological tools have allowed the introduction of multilevel hypernetworks, a powerful and sophisticated methodology capable of overcoming major limitations found in traditional analyses. ${ }^{7-9}$

Hypernetworks extend the concept of hypergraphs to model interactions of a set of elements (e.g., the players) that compose a given system (e.g., a football team). A hypergraph consists of a generalisation of a graph (a structure composed by a set of elements sharing some type of relation) in which an edge can connect any number of nodes (e.g., $\mathrm{n}$ players linked through spatial proximity). ${ }^{10}$ Hypernetworks comprise a natural multidimensional generalisation of networks and support representation of simultaneous $n$-ary relations by simplices with $n$ vertices (sub-groups of players in sports teams). ${ }^{10}$

A simplex consists of a generalisation of the notion of a triangle to arbitrary dimensions (e.g., 2-simplex (triangle); 3-simplex (tetrahedron)). In other words, a simplex consists of specific game structures (e.g., 2vs.1). Moreover, a simplex can be represented through a convex hull computation (the minimum convex area encompassing all players in the simplex) and encloses the velocity of each player (vector velocity regarding the instant $t-1$ and $t)$, as well as the velocity of the geometric centre of the simplices ${ }^{7}$ (see Figure 1).

The hyperedges of simplices are calculated using a proximity criterion, applied to each time frame of the match (i.e., by ascertaining the time evolution of simplices, that is: transformations of simplices through movements of players within and between simplices). Such a proximity criterion implicates that interactions between players, as well as sets of these interactions (i.e., simplices), may be evaluated through calculating and recording interpersonal distance values, such as spatial proximity and instant directional speed variables. $^{7}$ These relational variables capture whether an interaction between players occurs or not, and if a simplice can maintain its structure or not (i.e., disaggregated), when 
a player's velocity is moving away from the other players. Examples of previous studies using hypernetworks assessed interactions within and between simplices ${ }^{9}$ and examined the synchronisation tendencies emerging during competitive performance. ${ }^{8}$

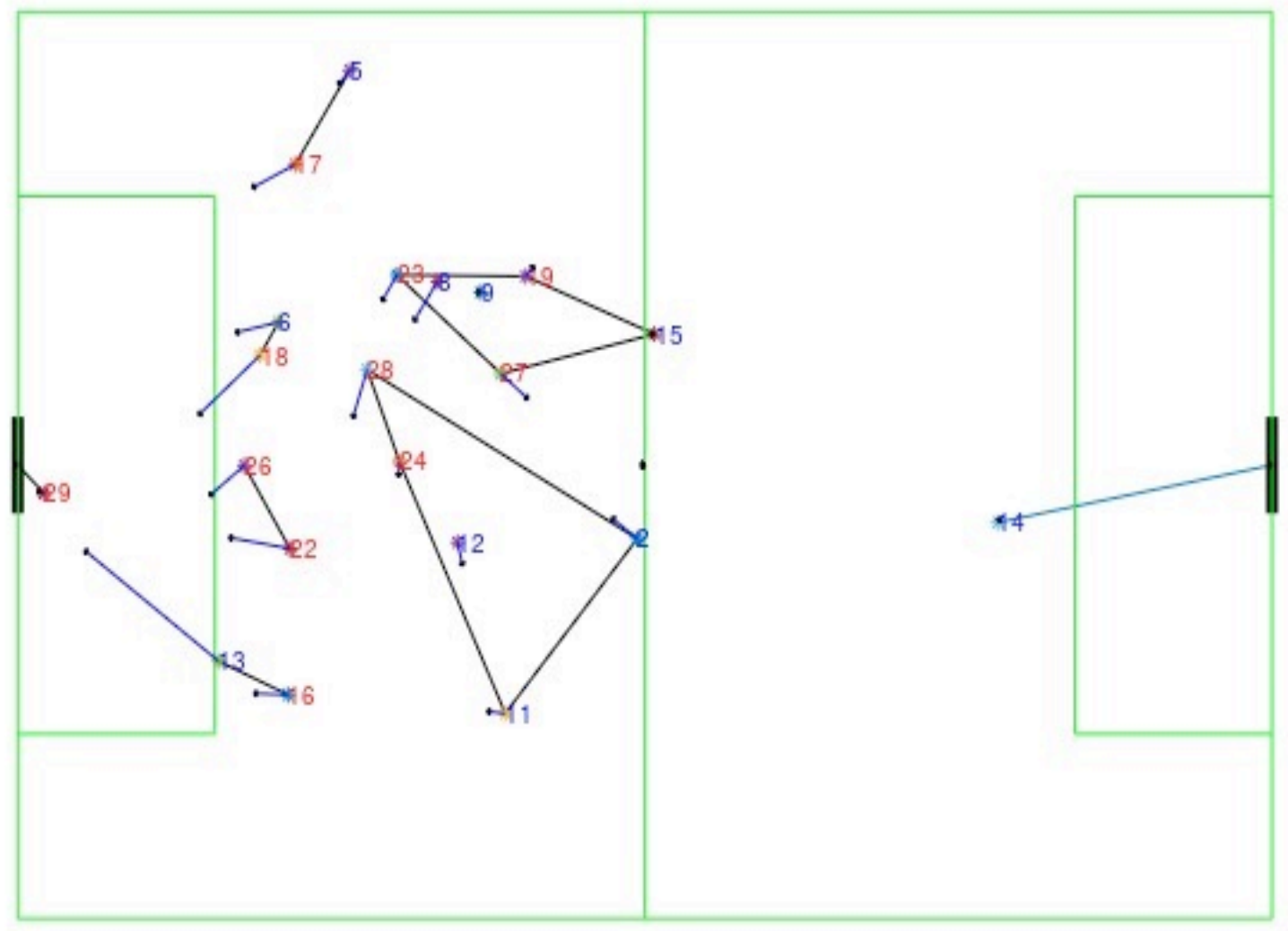

Figure 1. Schematic representation of players' simplices. Players composing the red team play from left to right, while players from the blue team play from right to left. Simplices formation is based on geographical proximity between players, with the arrows depicting players' direction of displacement. The goalkeepers (29- red team and 14 - blue team) are attached to their goals. There are four types of simplices, namely: 3 times $1 \mathrm{vs} .1$ composed of players 17 and 5, 18 and 6,16 and 13 from the red and blue team, respectively. $2 v s .0$ composed of players 26 and 22 from the red team. 2vs. 3 composed of players 28 and 24 from the red team, and players 12,11 and 2 from the blue team. $3 \mathrm{vs} .3$ composed of players 23,27 and 19 from the red team, and players 8,9 and 15 from the blue team.

Overall, the assembly of vertices to make simplices are key for understanding dynamical interactions between agents coordinating performance in multilevel systems that underpin effective communication and coordination in sports teams. Regardless, hypernetworks consist of a brand-new topic for sports sciences and more research is still needed to reveal complex properties of teams during competition.

\section{REFERENCES}

1. Duarte R, Araújo D, Correia V, et al. Sport teams as superorganisms: implications of biological models for research and practice in team sports performance analysis. Sports Med. 2012; 42(8): 633-42. doi: 10.2165/11632450-000000000-00000 
2. Gross T, Blasius B. Adaptive coevolutionary networks: a review. J R Soc Interface. 2008; 5:259-271. doi: 10.1098/rsif.2007.1229

3. Ribeiro J, Davids K, Araújo D, et al. Exploiting bi-directional self-organizing tendencies in team sports: the role of the game model and tactical principles of play. Front Psychol. 2019; 10:2213. doi: 10.3389/fpsyg.2019.02213

4. Sparrowe R, Liden R, Wayne $S$, et al. Social networks and the performance of individuals and groups. Acad Manage J. 2001; 44(2):316-25. doi: 10.2307/3069458

5. Ribeiro J, Silva P, Duarte R, et al. Team sports performance analysed through the lens of social network theory: Implications for research and practice. Sports Med. 2017; 47(9):1689-1696. doi: 10.1007/s40279-017-0695-1

6. Ramos J, Lopes RJ, Marques P, et al. Hypernetworks reveal compound variables that capture cooperative and competitive interactions in a soccer match. Front Psychol. 2019; 8:1379. doi: 10.3389/fpsyg.2017.01379

7. Ribeiro J, Davids K, Araújo D, et al. The role of hypernetworks as a multilevel methodology for modelling and understanding dynamics of team sports performance. Sports Med. 2019; 49:1337-1344. doi: 10.1007/s40279-019-01104-x

8. Ribeiro J, Lopes RJ, Silva P, et al. A multilevel hypernetworks approach to capture mesolevel synchronisation processes in football. J Sports Sci. 2019; 26:1-9. doi: 10.1080/02640414.2019.1707399

9. Ribeiro J, Silva P, Davids K, et al. A multilevel hypernetworks approach to capture properties of team synergies at higher complexity levels. Eur J Sport Sci. 2020; 4:1-11. doi: 10.1080/17461391.2020.1718214

10. Johnson J. Hypernetworks for reconstructing the dynamics of multilevel systems. In: European Conference on Complex Systems. Oxford, U.K; 2006.

Citation: Ribeiro J, Garganta J, Davids K, Barreira D. A multilevel hypernetworks approach to assess coordination and communication in player interactions in sports teams as co-evolutionary networks. BJMB. 2020: 14(5):167-170. Editors: Dr Fabio Augusto Barbieri - São Paulo State University (UNESP), Bauru, SP, Brazil; Dr José Angelo Barela São Paulo State University (UNESP), Rio Claro, SP, Brazil; Dr Natalia Madalena Rinaldi - Federal University of Espírito Santo (UFES), Vitória, ES, Brazil.

Guest Editors: Dr Rodrigo Aquino - Federal University of Espírito Santo (UFES), Vitória, ES, Brazil; Ms Luiz H Palucci Vieira - São Paulo State University (UNESP), Bauru, SP, Brazil; Dr Filipe Manuel Clemente - Escola Superior Desporto e Lazer, Instituto Politécnico de Viana do Castelo, Melgaço, Portugal; Dr João Cláudio Braga Pereira Machado -Federal University of Amazonas (UFAM), Manaus, AM, Brazil; Dr Gibson Moreira Praça - Universidade Federal de Minas Gerais (UFMG), Belo Horizonte, MG, Brazil.

Copyright: @ 2020 Ribeiro, Garganta, Davids and Barreira and BJMB. This is an open-access article distributed under the terms of the Creative Commons Attribution-Non Commercial-No Derivatives 4.0 International License which permits unrestricted use, distribution, and reproduction in any medium, provided the original author and source are credited.

Funding: There was no funding for this study.

Competing interests: The authors have declared that no competing interests exist.

DOI: https://doi.org/10.20338/bjmb.v14i5.216 